\title{
Mathematical Economics: Application of Fractional Calculus
}

\author{
Vasily E. Tarasov 1,2 (D) \\ 1 Skobeltsyn Institute of Nuclear Physics, Lomonosov Moscow State University, 119991 Moscow, Russia; \\ tarasov@theory.sinp.msu.ru; Tel.: +7-495-939-5989 \\ 2 Faculty "Information Technologies and Applied Mathematics", Moscow Aviation Institute (National \\ Research University), 125993 Moscow, Russia
}

Received: 22 April 2020; Accepted: 24 April 2020; Published: 27 April 2020

Mathematical economics is a theoretical and applied science in which economic objects, processes, and phenomena are described by using mathematically formalized language. In this science, models are formulated on the basis of mathematical formalizations of economic concepts and notions. An important purpose of mathematical economics is the formulation of notions and concepts in form, which will be mathematically adequate and self-consistent, and then, on their basis, to construct models of processes and phenomena of economy. The standard mathematical language, which is actively used in mathematical modeling of economy, is the calculus of derivatives and integrals of integer orders, the differential and difference equations. These operators and equations allowed economists to formulate models in mathematical form, and, on this basis, to describe a wide range of processes and phenomena in economy. It is known that the integer-order derivatives of functions are determined by the properties of these functions in an infinitely small neighborhood of the point, in which the derivatives are considered. As a result, economic models, which are based on differential equations of integer orders, cannot describe processes with memory and non-locality. As a result, this mathematical language cannot take into account important aspects of economic processes and phenomena.

Fractional calculus is a branch of mathematics that studies the properties of differential and integral operators that are characterized by real or complex orders. The methods of fractional calculus are powerful tools for describing the processes and systems with memory and nonlocality. There are various types of fractional integral and differential operators that are proposed by Riemann, Liouville, Grunwald, Letnikov, Sonine, Marchaud, Weyl, Riesz, Hadamard, Kober, Erdelyi, Caputo and other mathematicians. The fractional derivatives have a set of nonstandard properties such as a violation of the standard product and chain rules. The violation of the standard form of the product rule is a main characteristic property of derivatives of non-integer orders that allows us to describe complex properties of processes and systems.

Recently, fractional integro-differential equations are actively used to describe a wide class of economical processes with power-law memory and spatial nonlocality. Generalizations of basic economic concepts and notions of the economic processes with memory were proposed. New mathematical models with continuous time are proposed to describe the economic dynamics with a long memory.

The purpose of this Special Issue is to create a collection of articles reflecting the latest mathematical and conceptual developments in mathematical economics with memory and non-locality, based on applications of modern fractional calculus.

The proposed collection of works can be conditionally divided into three parts: historical, mathematical and applied.

This collection opens with two review articles, [1], by Vasily E. Tarasov, and [2], by Francesco Mainardi, purpose of which is a brief description of the history of the application of fractional calculus in economics and finance. 
The collection continues with a review work, [3], by Vasily E. Tarasov, the purpose of which is a description of the problems and difficulties arising in the construction of fractional-dynamic analogs of standard models by using fractional calculus. In article [4], by Anatoly N. Kochubei and Yuri Kondratiev, the authors proposed correct mathematical statements for growth models with memory in general cases, for application in mathematical economics of processes with memory and distributed lag. In article [5], by Jean-Philippe Aguilar, Jan Korbel and Yuri Luchko, applications of the fractional diffusion equation to option pricing and risk calculations are described. In work [6], by Jonathan Blackledge, Derek Kearney, Marc Lamphiere, Raja Rani and Paddy Walsh, authors discuss a range of results that are connected to Einstein's evolution equation, focusing on the Lévy distribution. In article [7], by Tomas Skovranek, a mathematical model, which is based on the one-parameter Mittag-Leffler function, is proposed to describe the relation between the unemployment rate and the inflation rate, also known as the Phillips curve. In article [8], by Yingkang Xie, Zhen Wang and Bo Meng, it is considered a fractional generalization of business cycle model with memory and time delay.

Further, this collection continues with works in which fractional calculus is applied to describe economy of different countries. In article [9], by José A. Tenreiro Machado, Maria Eugénia Mata and António M. Lopes, the fractional calculus and concept of pseudo-phase space are used for modeling the dynamics of world economies and forecasting a country's gross domestic product. In work [10], by Inés Tejado, Emiliano Pérez and Duarte Valério, the fractional calculus is applied to study the economic growth of the countries in the Group of Twenty (G20). In article [11], by Hao Ming, JinRong Wang and Michal Fečkan, the application of fractional calculus to economic growth models of Chinese economy is proposed. In work [12], by Ertuğrul Karaçuha, Vasil Tabatadze, Kamil Karaçuha, Nisa Özge Önal and Esra Ergün, the fractional calculus approach and the time series modeling are applied to describe the Gross Domestic Product (GDP) per capita for nine countries (Brazil, China, India, Italy, Japan, UK, USA, Spain and Turkey) and the European Union.

Funding: This research received no external funding.

Conflicts of Interest: The author declares no conflict of interest.

\section{References}

1. Tarasov, V.E. On History of Mathematical Economics: Application of Fractional Calculus. Mathematics 2019, 7, 509. [CrossRef]

2. Mainardi, F. On the Advent of Fractional Calculus in Econophysics via Continuous-Time Random Walk. Mathematics 2020, 8, 641. [CrossRef]

3. Tarasov, V.E. Rules for Fractional-Dynamic Generalizations: Difficulties of Constructing Fractional Dynamic Models. Mathematics 2019, 7, 554. [CrossRef]

4. Kochubei, A.N.; Kondratiev, Y. Growth Equation of the General Fractional Calculus. Mathematics 2019, 7, 615. [CrossRef]

5. Aguilar, J.-P.; Korbel, J.; Luchko, Y. Applications of the Fractional Diffusion Equation to Option Pricing and Risk Calculations. Mathematics 2019, 7, 796. [CrossRef]

6. Blackledge, J.; Kearney, D.; Lamphiere, M.; Rani, R.; Walsh, P. Econophysics and Fractional Calculus: Einstein's Evolution Equation, the Fractal Market Hypothesis, Trend Analysis and Future Price Prediction. Mathematics 2019, 7, 1057. [CrossRef]

7. Skovranek, T. The Mittag-Leffler Fitting of the Phillips Curve. Mathematics 2019, 7, 589. [CrossRef]

8. Xie, Y.; Wang, Z.; Meng, B. Stability and Bifurcation of a Delayed Time-Fractional Order Business Cycle Model with a General Liquidity Preference Function and Investment Function. Mathematics 2019, 7, 846. [CrossRef]

9. Tenreiro Machado, J.A.; Mata, M.E.; Lopes, A.M. Fractional Dynamics and Pseudo-Phase Space of Country Economic Processes. Mathematics 2020, 8, 81. [CrossRef]

10. Tejado, I.; Pérez, E.; Valério, D. Fractional Derivatives for Economic Growth Modelling of the Group of Twenty: Application to Prediction. Mathematics 2020, 8, 50. [CrossRef] 
11. Ming, H.; Wang, J.; Fečkan, M. The Application of Fractional Calculus in Chinese Economic Growth Models. Mathematics 2019, 7, 665. [CrossRef]

12. Karaçuha, E.; Tabatadze, V.; Karaçuha, K.; Önal, N.Ö.; Ergün, E. Deep Assessment Methodology Using Fractional Calculus on Mathematical Modeling and Prediction of Gross Domestic Product per Capita of Countries. Mathematics 2020, 8, 633. [CrossRef] 